\title{
Analysis of the photoproduction of massive gauge bosons at the LHeC
}

\author{
C. Brenner Mariotto ${ }^{1}$ and M. V.T. Machado ${ }^{2}$ \\ ${ }^{1}$ Instituto de Matematica, Estatistica e Fisica, Universidade Federal do Rio Grande, \\ Caixa Postal 474, CEP 96203-900, Rio Grande, Rio Grande do Sul, Brazil \\ ${ }^{2}$ High Energy Physics Phenomenology Group, GFPAE IF-UFRGS, \\ Caixa Postal 15051, CEP 91501-970, Porto Alegre, Rio Grande do Sul, Brazil
}

(Received 11 July 2012; published 21 August 2012)

\begin{abstract}
In this work we investigate the photoproduction of massive gauge bosons, $W^{ \pm}$and $Z^{0}$, as part of relevant physics topics to be studied in the proposed electron-proton collider, the Deep Inelastic ElectronNucleon Scattering at the $\mathrm{LHC}(\mathrm{LHeC})$. The estimates for production cross sections and the number of events are presented. In addition, motivated by the intensive studies to test the deviations from the Standard Model at present and future colliders, we discuss the $W^{ \pm}$asymmetries and perform an analysis on the role played by anomalous $W W \gamma$ coupling.
\end{abstract}

DOI: 10.1103/PhysRevD.86.033009

PACS numbers: 14.80.Bn, 12.60.-i, 13.85.Rm

\section{INTRODUCTION}

Being planned to start around 2020/2022, the Deep Inelastic Electron-Nucleon Scattering at the LHC (LHeC) machine is a possible extension of the current LHC at CERN, an electron-proton collider [1]. It is a convenient way to go beyond the LHC capabilities, exploiting the $7 \mathrm{TeV}$ proton beams that will be produced at the LHC, to drive research on $e p$ and $e A$ physics at some stage during the LHC time. This LHC extension will open a new kinematic window-the $\gamma p$ center-of-mass energy can reach up to $\mathrm{TeV}$ scale, far beyond the $\sqrt{s} \gg 200 \mathrm{GeV}$ at the Hadron-Electron Ring Accelerator at the DESY laboratory (HERA), a very profitable region for small- $x$ physics and many other physics studies. In particular, the energy of the incoming proton is delivered by the LHC beam, and a list of possible scenarios is considered for the energy of the incoming electron as $E_{p}=7 \mathrm{TeV}$ and $E_{e}=50-200 \mathrm{GeV}$, corresponding to the center of mass energies of $\sqrt{s}=$ $2 \sqrt{E_{p} E_{e}} \simeq 1.18-2.37 \mathrm{TeV}[2]$. The anticipated integrated luminosity is of order $10-10^{2} \mathrm{fb}^{-1}$ that depends on the energy of the electron beam and also the machine design.

Despite the great successes of the Standard Model (SM) and difficulties in finding new physics such as supersymmetry, which is the most popular scenario, the non-Abelian self-couplings of $W, Z$, and the photon remain poorly measured up to now. In this context, the investigation of three gauge boson couplings plays an important role to manifest the non-Abelian gauge symmetry in standard electroweak theory. Their precision measurement will be the crucial test of the structure of the SM. The inclusive and exclusive production of $W$ and $Z$ at the LHC already provides important tests of the SM and beyond. However, the photoproduction channel has the advantage of being much cleaner than the $p p$ collision channels. The physics program of the $\mathrm{LHeC}$ will explore the high-energy domain complementing the LHC and its discovery potential for physics beyond the SM with the great precision deep inelastic scattering measurements at high luminosities. The design report already contains [2] some estimates for a variety of electroweak interaction processes such as leptoquarks/leptogluons, new heavy leptons, new physics in boson-quark interactions, and sensitivity to a Higgs boson. In this work we investigate the photoproduction of massive gauge bosons at the $\mathrm{TeV}$ scale and also examine the potential of the $\mathrm{LHeC}$ collider to probe anomalous $W W \gamma$ coupling. Along these lines, we propose some observables that are sensitive to deviations from SM physics. Previous theoretical studies on such a subject are quite compelling, and the $W W \gamma$ vertex in $e p$ colliders was addressed in Refs. [3-6] long ago.

The aim of this work is twofold-first, we show predictions for the photoproduction of massive gauge bosons at future $\mathrm{LHeC}$ energies within SM physics. The photoproduction cross section including the resolved and direct processes are obtained, as well as their number of events in the most promising final state decays. We then go beyond it, with the photoproduction of $W$ bosons, analyzing the production rates of $W$ bosons, as they move away from the SM. The sensitivity of the LHC for deviations from the SM is investigated, and some additional observables are proposed. This article is organized as follows. The basic formulas to calculate the photoproduction of $W^{ \pm}, Z^{0}$, and virtual photons are presented in the next section, including the expressions for the anomalous coupling in the $W$-production case. Our numerical results for the photoproduction cross section and event rates within the SM and beyond are presented in Sec. III, followed by the corresponding discussion. The summary and conclusions are presented in Sec. IV.

\section{CROSS SECTIONS IN THE STANDARD MODEL AND BEYOND}

Let us start by considering the $C$ and $P$ parity conserving effective Lagrangian for two charged $W$-boson and one 
photon interactions [7]. The motivation is to use the $W$ photoproduction cross section as a test of the $W W \gamma$ vertex. In such a case, it is introduced by two dimensionless parameters, $\kappa$ and $\lambda$, which are related to the magnetic dipole and electric quadrupole moments, namely, $\mu_{W}=$ $\frac{e}{2 m_{W}}(1+\kappa+\lambda)$ and $Q_{W}=-\frac{e}{m_{W}^{2}}(\kappa-\lambda)$. In the case of values $\kappa=1$ and $\lambda=0$, the SM is recovered at tree level. We are left with three diagrams for the subprocess $\gamma q_{i} \rightarrow W q_{j}$ and only $t$ channel $W$ exchange graph contributes to the $W W \gamma$ vertex. The unpolarized differential cross section for the subprocess $\gamma q_{i} \rightarrow W q_{j}$ can be obtained using helicity amplitudes from summing over the helicities. For the signal, we are considering a quark jet and an on-shell $W$ with leptonic decay mode $\gamma p \rightarrow W^{+}+$jet $\rightarrow$ $\ell+p_{T}^{\text {miss }}+$ jet, where $\ell=e, \mu$. In the current mode, the charged lepton and the quark jet are nicely separated and the signal is prevented from being in the background of the SM.

The cross section for the subprocess $\gamma q_{i} \rightarrow W q_{j}$ is composed of the direct and resolved-photon production, $\hat{\sigma}=\hat{\sigma}_{\text {dir }}+\hat{\sigma}_{\text {res }}$. The direct-photon contribution is given by $[4,5]$

$$
\begin{aligned}
\hat{\sigma}_{W}= & \sigma_{0}\left\{| V _ { q _ { i } q _ { j } } | ^ { 2 } \left\{\left(\left|e_{q}\right|-1\right)^{2}\left(1-2 \hat{z}+2 \hat{z}^{2}\right) \log \left(\frac{\hat{s}-M_{W}^{2}}{\Lambda^{2}}\right)-\left[\left(1-2 \hat{z}+2 \hat{z}^{2}\right)-2\left|e_{q}\right|\left(1+\kappa+2 \hat{z}^{2}\right)\right.\right.\right. \\
& \left.+\frac{(1-\kappa)^{2}}{4 \hat{z}}-\frac{(1+\kappa)^{2}}{4}\right] \log \hat{z}+\left[\left(2 \kappa+\frac{(1-\kappa)^{2}}{16}\right) \frac{1}{\hat{z}}+\left(\frac{1}{2}+\frac{3\left(1+\left|e_{q}\right|^{2}\right)}{2}\right) \hat{z}\right. \\
& \left.+(1+\kappa)\left|e_{q}\right|-\frac{(1-\kappa)^{2}}{16}+\frac{\left|e_{q}\right|^{2}}{2}\right](1-\hat{z})-\frac{\lambda^{2}}{4 \hat{z}^{2}}\left(\hat{z}^{2}-2 \hat{z} \log \hat{z}-1\right) \\
& \left.+\frac{\lambda}{16 \hat{z}}(2 \kappa+\lambda-2)[(\hat{z}-1)(\hat{z}-9)+4(\hat{z}+1) \log \hat{z}]\right\},
\end{aligned}
$$

where $\sigma_{0}=\frac{\alpha G_{F} M_{W}^{2}}{\sqrt{2} \hat{s}}, \hat{z}=M_{W}^{2} / \hat{s}$ and $\Lambda^{2}$ is the cutoff scale in order to regularize the $\hat{u}$ pole of the collinear singularity for massless quarks. In addition, $\Lambda^{2}$ is the scale that determines the running of photon structure functions in the resolved part. The quantity $V_{i j}$ is the CabibboKobayashi-Maskawa (CKM) matrix, and $e_{q}$ is the quark charge.

The direct part of the cross section then reads

$$
\sigma_{\mathrm{dir}}\left(\gamma p \rightarrow W^{ \pm} X\right)=\int_{x_{p}^{m}}^{1} d x_{p} \sum_{q, \bar{q}} f_{q / p}\left(x_{p}, Q^{2}\right) \hat{\sigma}_{W}(\hat{s}),
$$

where $f_{q / p}$ are the parton distribution functions in the proton, $x_{p}^{m}=m_{W}^{2} / s$ and $\hat{s}=x_{p} s$.

The resolved-photon part of the cross section can be calculated using the usual electroweak formula for the $q_{\gamma} q_{p} \rightarrow W^{ \pm} \quad$ fusion process, $\quad \hat{\sigma}\left(q_{i} \bar{q}_{j} \rightarrow W\right)=$ $\frac{\sqrt{2} \pi}{3} G_{F} m_{W}^{2}\left|V_{i j}\right|^{2} \delta\left(x_{i} x_{j} s_{\gamma p}-m_{W}^{2}\right)$. For the photoproduction cross sections one needs parton distribution functions inside the photon and proton. The photon structure function $f_{q / \gamma}$ consists of perturbative pointlike parts and hadronlike parts. Putting it all together, the resolved-photon part reads

$$
\begin{aligned}
& \sigma_{\text {res }}\left(\gamma p \rightarrow W^{ \pm} X\right) \\
& =\frac{\pi \sqrt{2}}{3 s} G_{F} m_{W}^{2}\left|V_{i j}\right|^{2} \int_{x_{\gamma}^{m}}^{1} \frac{d x_{\gamma}}{x_{\gamma}} \sum_{q_{i}, q_{j}} f_{q_{i} / p}\left(\frac{m_{W}^{2}}{x s}, Q_{p}\right) \\
& \quad \times\left[f_{q_{j} / \gamma}\left(x_{\gamma}, Q_{\gamma}^{2}\right)-\tilde{f}_{q_{j} / \gamma}\left(x_{\gamma}, Q_{\gamma}^{2}\right)\right],
\end{aligned}
$$

where in order to avoid double counting on the leading logarithmic level, one subtracts the pointlike part of the photon structure function (photon splitting at large $x$ ), $\tilde{f}_{q / \gamma}\left(x, Q_{\gamma}^{2}\right)=\frac{3 \alpha e_{q}^{2}}{2 \pi}\left[x^{2}+(1-x)^{2}\right] \log \left(Q_{\gamma}^{2} / \Lambda^{2}\right)$. In addition, here $x_{\gamma}^{m}=m_{W}^{2} / s$.

Similar calculations can be done for the $Z$ boson photoproduction. Here, we focus on the SM prediction. Once again, the cross section for the subprocess $\gamma q \rightarrow Z q$ is composed of the direct and resolved-photon production, $\hat{\sigma}=\hat{\sigma}_{\text {dir }}+\hat{\sigma}_{\text {res }}$. The direct-photon contribution is given by

$$
\begin{aligned}
\hat{\sigma}_{Z}= & \frac{\alpha G_{F} M_{Z}^{2}}{\sqrt{2} \hat{s}} g_{q}^{2} e_{q}^{2}\left[\left(1-2 \hat{z}+2 \hat{z}^{2}\right) \log \left(\frac{\hat{s}-M_{Z}^{2}}{\Lambda^{2}}\right)\right. \\
& \left.+\frac{1}{2}\left(1+2 \hat{z}-3 \hat{z}^{2}\right)\right],
\end{aligned}
$$

where now $\hat{z}=M_{Z}^{2} / \hat{s}$ and $g_{q}^{2}=\frac{1}{2}\left(1-4\left|e_{e}\right| x_{W}+8 e_{q}^{2} x_{W}^{2}\right)$, with $x_{W}=0.23$.

The direct part of the $Z$-photoproduction cross section then reads

$$
\sigma_{\mathrm{dir}}\left(\gamma p \rightarrow Z^{0} X\right)=\int_{x_{p}^{m}}^{1} d x_{p} \sum_{q, \bar{q}} f_{q / p}\left(x_{p}, Q^{2}\right) \hat{\sigma}_{Z}(\hat{s}),
$$

where $f_{q / p}$ are the parton distribution functions in the proton, $x_{p}^{m}=m_{Z}^{2} / s$.

The resolved-photon part of the cross section stands for the subprocess $q \bar{q} \rightarrow Z^{0}$, and it is written as 


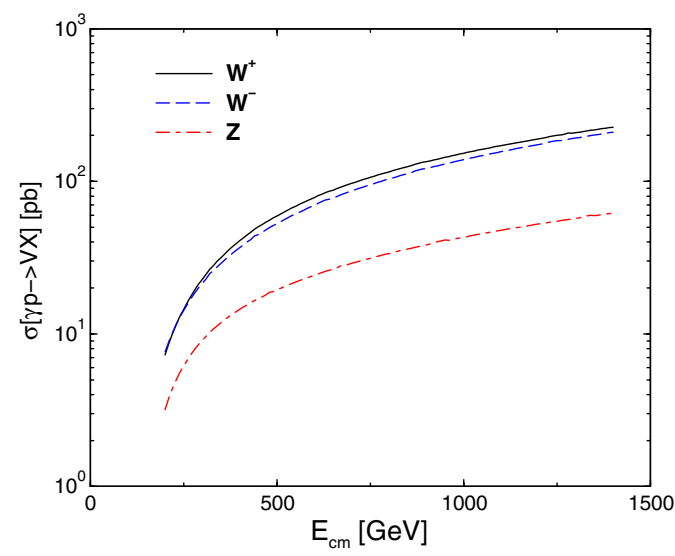

FIG. 1 (color online). Cross sections for the production of massive $W^{ \pm}$and $Z^{0}$ gauge bosons as a function of the centerof-mass energy.

$$
\begin{aligned}
& \sigma_{\text {res }}(\gamma p\left.\rightarrow Z^{0} X\right) \\
&= \frac{\pi \sqrt{2}}{3 s} G_{F} m_{W}^{2} g_{q}^{2} \int_{x_{\gamma}^{m}}^{1} \frac{d x_{\gamma}}{x_{\gamma}} \sum_{q} f_{\bar{q} / p}\left(\frac{m_{Z}^{2}}{x s}, Q_{p}\right) \\
& \quad \times\left[f_{q / \gamma}\left(x_{\gamma}, Q_{\gamma}^{2}\right)-\tilde{f}_{q / \gamma}\left(x_{\gamma}, Q_{\gamma}^{2}\right)\right] .
\end{aligned}
$$

In the next section we compute the numerical results for the $W^{ \pm}$and $Z^{0}$ photoproduction cross section in the LHeC regime of energy/luminosity. We also investigate the sensitivity to anomalous $W W \gamma$ couplings associated to beyond SM physics.

\section{NUMERICAL RESULTS AND DISCUSSIONS}

Let us now perform a preliminary study for the LHeC machine [1,2]. Using the design with an electron beam having laboratory energy of $E_{e}=70 \mathrm{GeV}$, the center of mass energy will reach $E_{\mathrm{cm}}=W_{\gamma p}=1.4 \mathrm{TeV}$ and a nominal luminosity of order $10^{33} \mathrm{~cm}^{-2} \mathrm{~s}^{-1}$. Our estimates for the massive boson photoproduction cross sections in the SM are the following. One gets $\sigma\left(\gamma+p \rightarrow W^{ \pm} X\right) \simeq$ $400 \mathrm{pb}$ and $\sigma\left(\gamma+p \rightarrow Z^{0} X\right) \simeq 60 \mathrm{pb}$. These are roughly estimates, since we have not introduced the $K$ factors associated with next-to-leading-order (NLO) corrections to the processes. We have summed the resolved and direct contributions. The energy behavior for the cross section is presented in Fig. 1. The dependence is quantitatively given

TABLE I. The photon-proton cross sections times branching ratios $\sigma\left(\gamma p \rightarrow W^{ \pm} X\right) \times \mathrm{BR}\left(W^{+} \rightarrow \mu \nu\right)$ and $\sigma\left(\gamma p \rightarrow Z^{0} X\right) \times$ $\operatorname{BR}\left(Z^{0} \rightarrow \mu^{+} \mu^{-}\right)$in units of pb. The number of events $N_{\mathrm{ev}}$ is also presented at an integrated luminosity of $10 \mathrm{fb}^{-1}$.

\begin{tabular}{lcc}
\hline \hline$V$ & $\sigma(\gamma p \rightarrow V X) \times \mathrm{BR}$ & $N_{\mathrm{ev}}$ \\
\hline$W^{+}$ & 24 & $1.2 \times 10^{4}$ \\
$W^{-}$ & 24 & $1.2 \times 10^{4}$ \\
$Z^{0}$ & 2.1 & $1.1 \times 10^{3}$ \\
\hline \hline
\end{tabular}

by $\sigma_{V} \propto W_{\gamma p}^{\alpha}$, with $\alpha \simeq 1.312$. It is seen that the cross sections are at least 1 order of magnitude larger than for the DESY-HERA machine, $W_{\gamma p} \simeq 300 \mathrm{GeV}$.

In Table I the photon-proton total cross sections times branching ratio of $W \rightarrow \mu \nu$ and the corresponding number of events are shown for SM parameters for $W(\kappa=1$ and $\lambda=0$ ) and also for the $Z^{0}$ boson with a corresponding branching ratio of $Z^{0} \rightarrow \mu^{+} \mu^{-}$. The number of events has been computed using $N_{\mathrm{ev}}=\sigma(e p \rightarrow V+X) \times$ $\operatorname{BR}\left(V \rightarrow \mu \nu / \mu^{+} \mu^{-}\right) L_{\text {int }}$. At this point we consider the acceptance in the leptonic channel as $100 \%$. The photoproduction cross section is calculated by convoluting the Weizsäcker-Williams spectrum

$$
\begin{aligned}
f_{\gamma / e}(y)= & \frac{\alpha}{2 \pi}\left[\frac{1+(1-y)^{2}}{y} \log \frac{Q_{\max }^{2}}{Q_{\min }^{2}}\right. \\
& \left.-2 m_{e}^{2} y\left(\frac{1}{Q_{\min }^{2}}-\frac{1}{Q_{\max }^{2}}\right)\right],
\end{aligned}
$$

with the differential hadronic cross section. Here, $Q_{\min }^{2}=$ $m_{e}^{2} y /(1-y)$ and we impose a cut of $Q_{\min }^{2}=0.01$.

Through the calculations, proton structure functions of CTEQ [8] and photon structure functions of Glück-ReyaVogt [9] have been used with $Q^{2}=M_{W}^{2}$. The usual electroweak parameters are taken from Ref. [10]. We have assumed an integrated luminosity $L_{\text {int }}$ at $10 \mathrm{fb}^{-1}$ [2] in order to compute the number of events, $N_{\mathrm{ev}}$. The number of events is large enough to put forward further analysis, as we have units of events per second for $W^{ \pm}$.

Let us now investigate the scenario for physics beyond the SM. Certain properties of the $W$ bosons, such as the magnetic dipole and the electric quadrupole moment, play a role in the interaction vertex $W W \gamma$; thus processes involving this vertex offer the opportunity to measure such properties. The magnetic dipole moment $\mu_{W}$ and the electric quadrupole moment $Q_{W}$ of the $W$ bosons can be written in terms of parameters $\kappa, \lambda$, where $\kappa=1$ and $\lambda=0$ are the Standard Model values for those parameters at tree level. According to the Particle Data Group [10], the measured value of $\mu_{W} / \frac{e}{2 M_{W}}=1+\kappa+\lambda=2.22 \pm 0.20$ suggests that there are deviations from the standard values. In $W$ photoproduction one has a unique scenario to test the anomalous $W W \gamma$ vertex and its $\kappa$ and $\lambda$ parameters. The $W W \gamma$ vertex $\left[W^{+}\left(p_{1}\right), W^{-}\left(p_{2}\right), A\left(p_{3}\right)\right]$, denoted by $\Gamma_{\mu \nu \rho}\left(p_{1}, p_{2}, p_{3}\right)$, is given by [11]

$$
\begin{aligned}
\frac{\Gamma_{\mu \nu \rho}}{e}= & {\left[g_{\mu \nu}\left(p_{1}-p_{2}-\frac{\lambda}{M_{W}^{2}}\left[\left(p_{2} \cdot p_{3}\right) p_{1}-\left(p_{1} \cdot p_{3}\right) p_{2}\right]\right)_{\rho}\right.} \\
& +g_{\mu \rho}\left(\kappa p_{3}-p_{1}+\frac{\lambda}{M_{W}^{2}}\left[\left(p_{2} \cdot p_{3}\right) p_{1}-\left(p_{1} \cdot p_{2}\right) p_{3}\right]\right)_{\nu} \\
& +g_{\nu \rho}\left(p_{2}-\kappa p_{3}-\frac{\lambda}{M_{W}^{2}}\left[\left(p_{1} \cdot p_{3}\right) p_{2}-\left(p_{1} \cdot p_{2}\right) p_{3}\right]\right)_{\mu} \\
& \left.+\frac{\lambda}{M_{W}^{2}}\left(p_{2 \mu} p_{3 \nu} p_{1 \rho}-p_{3 \mu} p_{1 \nu} p_{2 \rho}\right)\right],
\end{aligned}
$$


TABLE II. The number of muon plus neutrino events coming from the $W^{+}$decay for distinct choices for the parameters $\kappa$ and $\lambda$ presented at an integrated luminosity of $10 \mathrm{fb}^{-1}$.

\begin{tabular}{cccc}
\hline \hline$\kappa$ & $\lambda$ & $\sigma\left(\gamma p \rightarrow W^{+} X\right) \times \mathrm{BR}[\mathrm{pb}]$ & $N_{\mathrm{ev}}$ \\
\hline 0 & 0 & 16 & $8 \times 10^{3}$ \\
1 & 0 & 24 & $1.2 \times 10^{4}$ \\
2 & 0 & 44 & $2.2 \times 10^{4}$ \\
1 & 1 & 61 & $3.1 \times 10^{4}$ \\
1 & 2 & 172 & $8.5 \times 10^{4}$ \\
\hline \hline
\end{tabular}

where the anomalous contributions from the SM are taken into account if they are included in the terms involving $\kappa \neq 1$ and/or $\lambda \neq 0$.

In the photoproduction of $W^{ \pm}$bosons, the direct contribution $\sigma_{\text {dir }}$ involves the generalized $W W \gamma$ vertex, and then the expression for $\hat{\sigma}_{W}\left(\hat{s}=x_{p} s\right)$ in Eq. (1) can be used to investigate deviations from SM physics. An interesting observable is the number of muon plus neutrino events coming from the decay of the $W^{+}$. This is shown in Table II, where we assumed the luminosity of $\mathcal{L}=$ $10 \mathrm{fb}^{-1}$. As we can see, the number of $W^{+} \rightarrow \mu \nu$ events is very dependent on the choice of the $\kappa$ and $\lambda$ parameters, and in most scenarios it increases as $\kappa, \lambda$ increase/depart from the Standard Model. Such an effect could certainly be tested at the LHeC.

As we have already noticed, the cross sections for the $W$ and $Z$ production may have contributions due to higherorder terms that where not included in our calculation. Cross sections in NLO considering the default Standard Model vertices were already calculated in Ref. [12]. In order to get rid of normalization uncertainties, one can take the ratios $\sigma_{W}^{ \pm} / \sigma_{Z}$ to test the $W W \gamma$ vertex and the $\kappa$ and $\lambda$ parameters. To do this, we propose the study of the following observable:

$$
R_{W / Z}(\kappa, \lambda ; \sqrt{s})=\frac{\sigma_{W^{+}}+\sigma_{W^{-}}}{\sigma_{Z}},
$$

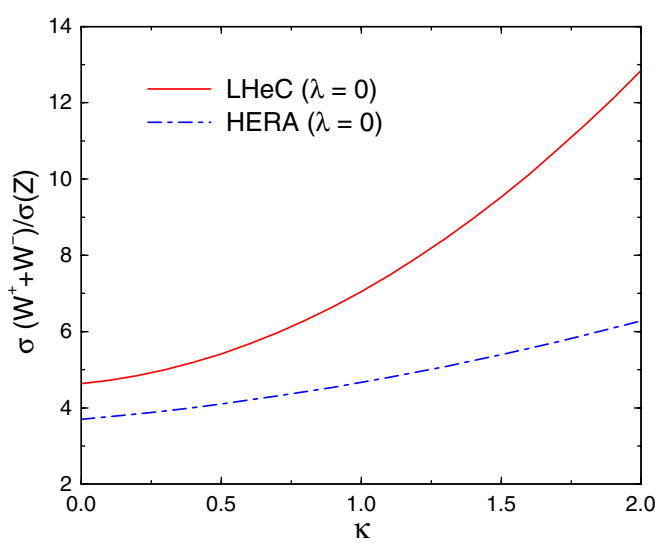

which can be constructed from equations given in the previous section. Such an observable was already proposed some time ago, in Refs. [4,5]. In Fig. 2 we show our results for the $R_{W / Z}$ ratio, for HERA and LHeC energies. In the left plot, the dependence of the ratio is shown as a function of the $\kappa$ parameter for fixed $\lambda=0$. In addition, in the right plot the ratio is presented as a function of the $\lambda$ parameter for fixed $\kappa=1$. The results are presented for both DESYHERA and LHeC energies. We can study the sensitivity with $\kappa$ and $\lambda$ parameters, regarding the SM and possible new physics. The results show that the ratio has much more sensitivity to the $\kappa$ and $\lambda$ parameters for $\mathrm{LHeC}$ energies. Regarding the $\lambda$ parameter, it is unimportant at HERA energies. Thus, the LHeC collider would be able to pin down the correct values for these parameters and then determine the magnet dipole and electric quadrupole of the $W$.

Another observable that could be studied and tested at the $\mathrm{LHeC}$ is the $W^{+} W^{-}$asymmetry, defined by

$$
A(\kappa, \lambda ; \sqrt{s})=\frac{\left(\sigma_{W^{+}}-\sigma_{W^{-}}\right)}{\left(\sigma_{W^{+}}+\sigma_{W^{-}}\right.} .
$$

The results for this asymmetry are shown in Fig. 3, where we show the sensitivity of the $\kappa$ and $\lambda$ parameters. As we can see, for $\mathrm{LHeC}$ energies the $W^{+} W^{-}$asymmetry depends strongly on the $\kappa$ and $\lambda$ parameters and is therefore a useful observable to help determine the best scenarios. In the left plot we show the dependence of asymmetry as a function of the $\kappa$ parameter for fixed $\lambda=0$ (its SM value) only for the $\mathrm{LHeC}$ energy. Moreover, in the right plot we present the ratio as a function of the $\lambda$ parameter for fixed $\kappa=1$, where the corresponding result for DESYHERA energy is also shown. As a general conclusion about the anomalous coupling, we see that with the LHeC collider the parameters $\kappa$ and $\lambda$ have better sensitivity than the DESY-HERA $e p$ collider, and it would give complementary information to the LHC collider.

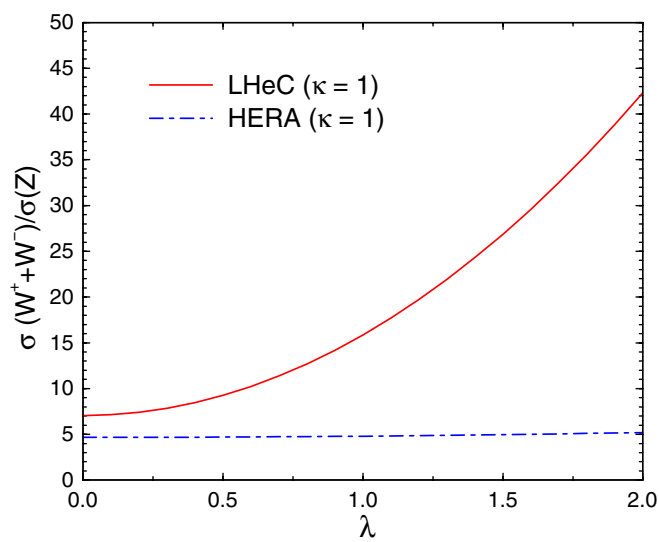

FIG. 2 (color online). The ratio $R_{W / Z}(\kappa, \lambda)$ for both DESY-HERA and LHeC energies. Left panel: ratio as a function of the $\kappa$ parameter for fixed $\lambda=0$ (SM value). Right panel: ratio as a function of the $\lambda$ parameter for fixed $\kappa=1$ (SM value). 

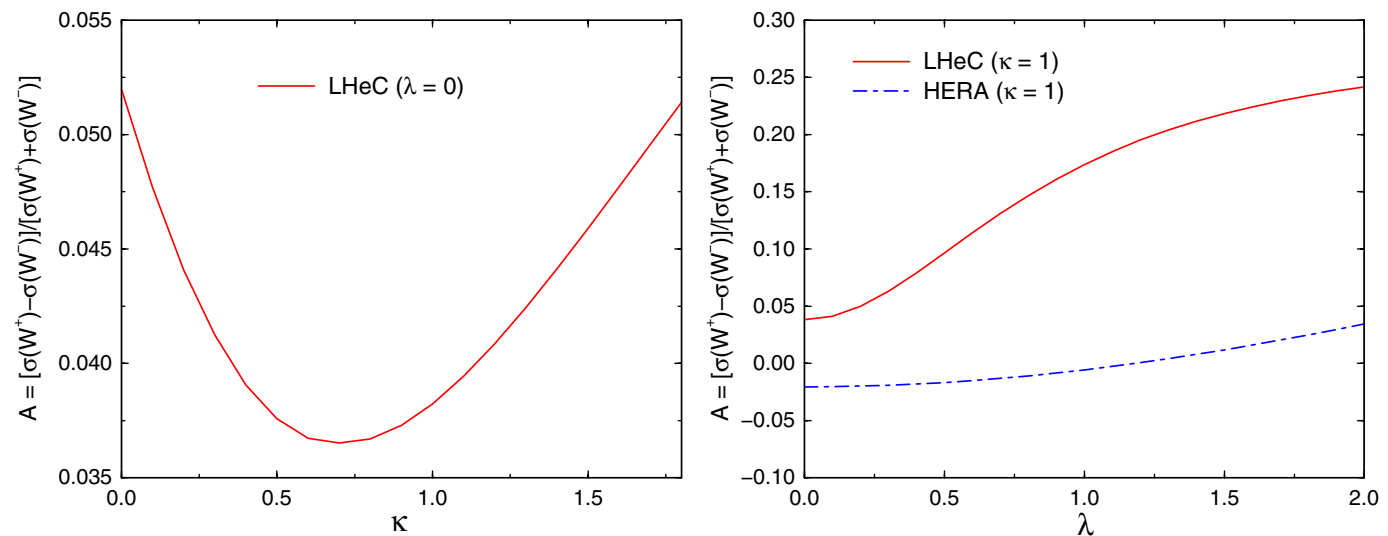

FIG. 3 (color online). The asymmetry $A(\kappa, \lambda ; \sqrt{s})$ at the LHeC regime. Left panel: asymmetry as a function of the $\kappa$ parameter for fixed $\lambda=0$ (SM value). Right panel: asymmetry as a function of the $\lambda$ parameter for fixed $\kappa=1$ (SM value). In this last case, the result for DESY-HERA energy is also shown.

Finally, let us compare the present calculation to previous studies. In Ref. [5] the massive boson photoproduction is considered for an energy of $\sqrt{s} \simeq 1.3 \mathrm{TeV}$ and integrated luminosity of $1 \mathrm{fb}^{-1}$. For the SM values of $\kappa$ and $\lambda$ parameters, the photoproduction cross section was obtained as $11.3,12.2$, and $5.4 \mathrm{pb}$ for $W^{+}, W^{-}$, and $Z^{0}$, respectively. The number of events for $Z^{0}$ was estimated to be 360 . These results are completely consistent with ours when considering the integrated luminosity of $10 \mathrm{fb}^{-1}$. Concerning the sensitivity to the parameters, we found the general trend is similar; however, the cross sections for higher values of parameters are relatively larger than ours. We have checked that our ratio $\sigma\left(W^{ \pm}\right) / \sigma(Z)$ is $50 \%$ smaller than in Ref. [5] for several values of parameters $\kappa$, $\lambda$. This probably is due to the different energy and the theoretical uncertainties coming from the parton distribution functions considered.

In Ref. [6] an analysis quite similar to ours was performed focusing on the spectrum of the backscattered laser photon (energy of $\sqrt{s}=1.7 \mathrm{TeV}$ and integrated luminosity of $200 \mathrm{pb}^{-1}$ ). For a Weizsäcker-Williams spectrum, we have checked that the numbers of events in the process $\gamma p \rightarrow W^{+}$jet is quite consistent with ours when considering the same integrated luminosity. Their original values are, for instance, 288 and 1151 events for sets $(\kappa=1$, $\lambda=0)$ and ( $\kappa=1, \lambda=2)$, respectively. The compatibility is good, as we are using a luminosity 100 times larger.

The photoproduction of the $W$ boson at HERA has been addressed at the NLO level of QCD corrections in Ref. [12]. The prediction is $\sigma\left(W^{+}\right)=0.478 \mathrm{pb}$ and $\sigma\left(W^{-}\right)=0.484 \mathrm{pb}$ at $\sqrt{s}=318 \mathrm{GeV}$, imposing a cut $p_{T}<25 \mathrm{GeV}$. The main conclusion is that the QCD corrections reduce the factorization scale dependence significantly and modify the leading-order prediction by a factor of $10 \%$. This can be used as a good argument for our LO calculations here. In addition, we can rescale their prediction for the $\mathrm{LHeC}$ case. A rough estimate would give
$\sigma\left(W^{+}\right) \times \mathrm{BR}=0.33 \mathrm{pb}$ and $\sigma\left(W^{-}\right) \times \mathrm{BR}=0.34 \mathrm{pb}$ at the LHeC. This is smaller than our results in Table I, where one has $\sigma\left(e+p \rightarrow W^{ \pm}+X\right) \times \mathrm{BR}=1.2 \mathrm{pb}$, which is associated with the cut on boson transverse momentum and distinct kinematic cuts in the integration of the Weizsäcker-Williams spectrum.

\section{SUMMARY}

We have examined the prospects for massive gauge bosons detection at the proposed Deep Inelastic ElectronNucleon Scattering at the LHC (LHeC) machine. The photon-proton cross sections have been computed for $W^{ \pm}$and $Z^{0}$ inclusive production and are of the order of dozens of picobarns. The number of events is evaluated for the photoproduction cross section, assuming an integrated luminosity of $10 \mathrm{fb}^{-1}$ and, they are large enough to make the measurements feasible. We have also investigated the anomalous $W W \gamma$ coupling using the machine design. We found that the likelihood of that kinematic limit to be available at the $\mathrm{LHeC}$ is somewhat increased relative to the previous DESY-HERA machine. We have tested some sample scenarios beyond SM physics by scanning the values of parameters $\kappa$ and $\lambda$ considering anomalous $W W \gamma$ coupling. In the case of anomalous coupling, the photoproduction process at the $\mathrm{LHeC}$ proves to be a powerful tool. Finally, we consider different observables that together could contribute to pinning down the correct $W W \gamma$ vertex. We introduced the ratio $\sigma\left(W^{ \pm}\right) / \sigma(Z)$, which is less sensitive to the NLO QCD corrections and the $W$-asymmetry observable $A(\kappa, \lambda ; \sqrt{s})$ that scans asymmetries in the $W$ photoproduction.

\section{ACKNOWLEDGMENTS}

This research was supported by CNPq and FAPERGS, Brazil. 
[1] J. B. Dainton, M. Klein, P. Newman, E. Perez, and F. Willeke, JINST 1, P10001 (2006).

[2] O. Bruening, M. Klein, and P. Laycock (LHeC Study Group Collaboration), arXiv:1206.2913.

[3] U. Baur and D. Zeppenfeld, Nucl. Phys. B325, 253 (1989).

[4] C.S. Kim and W. J. Stirling, Z. Phys. C 53, 601 (1992).

[5] C. S. Kim, J. Lee, and H.S. Song, Z. Phys. C 63, 673 (1994).

[6] S. Atag and I. T. Cakir, Phys. Rev. D 63, 033004 (2001).
[7] K. Hagiwara, R. D. Peccei, D. Zeppenfeld, and K. Hikasa, Nucl. Phys. B282, 253 (1987).

[8] J. Pumplin, D. R. Stump, J. Huston, H. L. Lai, P. Nadolsky, and W. K. Tung, J. High Energy Phys. 07 (2002) 012.

[9] M. Glück, E. Reya, and A. Vogt, Phys. Rev. D 45, 3986 (1992).

[10] K. Nakamura et al. (Particle Data Group), J. Phys. G 937, 0755021 (2010).

[11] M. N. Dubinin and H.S. Song, Phys. Rev. D 57, 2927 (1998).

[12] K.-P. O. Diener, Ch. Schwanenberger, and M. Spira, Eur. Phys. J. C 25, 405 (2002). 